\title{
Operatoriedade cognitiva e experimentação virtual imersiva de Eletricidade
}

\section{Cognitive Operativeity and Immersive Virtual Experimentation of Electricity}

\author{
Valter A. Ferreira - PPGIE - UFRGS - valter.unipampa@gmail.com \\ Andreia S. Bos - PPGIE - UFRGS - andreia.bos@gmail.com \\ Liane M. R. Tarouco - PPGIE - UFRGS - liane@ penta.ufrgs.br \\ Fernando Becker - PPGIE - UFRGS - fbeckerufrgs@gmail.com
}

\begin{abstract}
Resumo: Este trabalho empregou a plataforma virtual imersiva 3D OpenSim, para o desenvolvimento de um experimento sobre Eletricidade. A Epistemologia Genética e a teoria da Abstração Reflexionante de Piaget foram o pano de fundo, tanto para o projeto do circuito, quanto para a coleta e análise dos dados. A gravação audiovisual da apliação da entrevista clínica em conjunto com as simulações virtuais, permitiu a coleta de informações comportamentais e de desempenho dos sujeitos. Os dados foram transcritos em protocolos, conforme o Método Clínico e a análise revelou a adequação do uso da teoria de Piaget para a criação de experimentos virtuais imersivos 3D de Eletricidade. Ademais, os sujeitos da pesquisa demonstraram o uso do pensamento formal frente às situações-problema apresentadas pelas simulações computacionais.
\end{abstract}

Palavras-chave: Laboratório Virtuais Imersivos 3D; Epistemologia Genética; Ensino de Física.

Abstract: This work employed the OpenSim 3D immersive virtual platform for the development of an Electricity experiment. Genetic Epistemology and Piaget's Theory of Reflective Abstraction were the backdrop for both circuit design and data collection and analysis. The audiovisual recording of the clinical interview application in conjunction with the virtual simulations, allowed the collection of behavioral and performance information of the subjects. Data were transcribed into protocols according to the Clinical Method and the analysis revealed the appropriateness of using Piaget's theory for the creation of immersive 3D Electricity experiments. Moreover, the research subjects demonstrated the use of formal thinking in face of problem situations presented by computer simulations.

Keywords: Virtual Immersive 3D Laboratory; Genetic Epistemology; Physics Teaching.

\section{INTRODUÇÃO}

O Eletromagnetismo faz parte da vida cotidiana mais do que se imagina, através dele é possível compreender, entre outros fenômenos naturais, a coesão entre os átomos e as formações do relâmpago, do arco-íris e da aurora boreal. Além disto, tais princípios científicos serviram de base para a invenção de lâmpadas, aparelhos de rádio, receptores de televisão, micro-ondas, computadores, celulares, etc (HALLIDAY, RESNICK E WALKER, 2016).

A importância deste ramo científico é reconhecida pelos Parâmetros Curriculares Nacionais para o Ensino Médio (BRASIL, 2006), os quais incentivam o desenvolvimento de práticas pedagógicas voltadas à construção de conceitos eletromagnéticos, por considerá-los fundamentais para uma adequada leitura de Mundo. No entanto, esta área 
ainda é qualificada por alunos e professores como de difícil compreensão, envolvendo uma complexa abordagem teórico-matemática que, em geral, desconsidera o auxílio da prática experimental.

Esse cenário preocupante tem levado pesquisadores a criação de estratégias para o aprimoramento do ensino de Eletromagnetismo, como a proposta por Guedes (2017), que desenvolveu uma série de experimentos de baixo custo objetivando dirimir o alto grau de abstração requerido para o aprendizado do conceito de linhas de campo magnético, campo magnético criado por corrente elétrica e força sobre um condutor imerso em campo magnético. Mentz (2017), por sua vez, observando a falta de conhecimento matemático, a baixa motivação e o desconhecimento da importância dos conceitos de ondas eletromagnéticas, sugeriu uma sequência didática de aprendizagem por pesquisa com $o$ uso de celulares.

Santos (2016) partiu de princípios físicos responsáveis pela integração entre Eletricidade e Magnetismo, mas de difícil visualização (campo magnético no espaço tridimensional), para desenvolver um laboratório virtual acessado via internet (LABVIRT), destinado ao estudo de tópicos de Eletromagnetismo com base na Teoria dos Campos Conceituais ${ }^{1}$ de Vergnaud.

A conjuntura das dificuldades de aprendizagem conceitual, somada ao fato do Eletromagnetismo não ter sido abordado nos estudos de Piaget e Inhelder (1976) (somente Ótica, Hidrostática e Mecânica) levaram à escolha de investigar o processo de construção de conhecimento envolvendo este campo da Física e a estratégia de criação do experimento levou em conta situações de equilíbrio físico com base nas relações do grupo INRC $^{2}$.

A facilidade de criação e adaptação às especificidades dos sujeitos, além de potencializar sensorialmente a experiência virtual, promoveram o desejo de trabalhar com uma plataforma virtual imersiva 3D e a opção pela Epistemologia Genética de Jean Piaget justifica-se por esta fornecer elementos para investigar a sequência de pensamento do sujeito e como este se reequilibra cognitivamente frente à resistência oferecida pelo objeto de conhecimento (no caso, o experimento virtual proposto).

Em suma, aliando o potencial de construção de conhecimento da plataforma OpenSin com a abordagem de compreensão do processo de equilibração cognitiva do sujeito da Epistemologia Genética de Jean Piaget, o presente artigo apresenta a seguinte questão de pesquisa: Qual o esforço operatório de pensamento, exigido pela simulação de um experimento virtual imersivo 3D sobre a lei de Kirchoff, desenvolvido com base no grupo INRC de Piaget?

\section{EPISTEMOLOGIA GENÉTICA}

A obra do psicólogo suíço Jean Piaget (1896-1980) é uma importante referência para estudos sobre a construção de conhecimento, pois aborda o sujeito epistêmico como o indivíduo criador do seu saber por força de sua ação sobre o Mundo ao redor. Para Piaget (1977) a elaboração conceitual não parte do zero, não principia no sujeito ou no objeto ou configura mero registro, mas é fruto de uma estrutura em constante diferenciação. Para a Epistemologia Genética, o conhecimento não é inerente ao próprio sujeito (apriorismo), nem advém unicamente das observações do meio (empirismo), mas é construído pela

\footnotetext{
${ }^{1}$ A Teoria dos Campos Conceituais descreve como os alunos constroem o conhecimento matemático, no qual a aprendizagem acontece por meio de experiências envolvendo um grande número de situações, tanto dentro como fora da escola.

${ }^{2} \mathrm{O}$ grupo de quatro transformações INRC (Identidade, Negação, Recíproca e Correlação) é a fusão, em um sistema único, das duas formas de reversibilidade (inversão ou negação e reciprocidade), formando uma estrutura completa e fechada, característica de sistemas em equilíbrio físico.
} 
interação do sujeito com o meio, levando em conta as estruturas cognitivas já existentes. Ela preconiza que a construção do conhecimento de " $x$ " para " $x+1$ ", não ocorre da mesma forma para todos os sujeitos, pois depende da mútua influência de quatro fatores (MORAES, 2017):

a) Hereditariedade e maturação do sistema nervoso central: condição necessária, mas insuficiente para a evolução do conhecimento, responsável pela ordem de sucessão dos estádios de desenvolvimento e pelas primeiras aprendizagens;

b) Experiências físicas e lógico-matemáticas: exprimem a interação sujeito-objeto, a primeira relaciona-se à ação do sujeito sobre o objeto e a segunda à coordenação destas ações, sejam elas reais ou representadas;

c) Transmissão social: Fator educativo cuja eficácia está atrelada a um processo ativo de assimilação pelo sujeito;

d) Equilibração das estruturas cognitivas: Ápice do processo interativo de aprendizado, condicionado ao equilíbrio entre os três fatores anteriores e cuja representação ocorre pela compensação ativa dos desequilíbrios advindos da resistência do objeto às tentativas de assimilação realizadas pelo sujeito.

A inexistência de uniformidade na hereditariedade, nas experiências e nas interações sociais vividas por cada sujeito, implica em um progresso cognitivo não linear e considera a construção da capacidade de conhecer a partir de um nível mais elementar para outro de maior complexidade. Existem quatro grandes estádios de desenvolvimento: SensórioMotor (0 a 2 anos), Pré-operatório (2 a 7 anos), Operatório-Concreto (7 a 11 anos), Operatório-Formal (12 anos em diante), nos quais a passagem para o subsequente depende da superação do anterior, os níveis inferiores constroem os superiores, podem ser diferenciados pelos processos de formação e pelas formas finais de equilíbrio e a fronteira entre os estádios inicia quando as características de ambos se misturam (OBANA, 2015).

Ao longo destes estádios, a necessidade de adaptar-se ao mundo ao redor faz o sujeito agir sobre os objetos e integrar os novos estímulos às suas estruturas prévias ou esquemas $^{3}$, configurando o processo de assimilação, cuja importância reside no fato de todo conhecimento referir-se tanto à significações quanto à ação de conhecer um objeto, o qual é assimilado aos esquemas de ação através de condutas sensório-motoras até chegar às operações lógico-matemáticas superiores. No entanto, quando o sujeito defronta-se com um novo estímulo que não consegue assimilar, surge a necessidade de modificar a estrutura cognitiva prévia ou criar uma nova. Esta alteração de esquemas ou estruturas cognitivas de assimilação, sob influência de estímulos externos, é chamada de acomodação. Tal processo caracteriza-se pela a capacidade de antecipação, podendo prever uma quantidade de acontecimentos e um conjunto de transformações possíveis, mostrando a potência dedutiva do pensamento (MORAES, 2017).

O ciclo assimilação $\leftrightarrow$ acomodação desencadeia o processo de equilibração majorante, evidenciado pelos desequilíbrios oriundos da tentativa de solucionar uma situaçãoproblema, cujas reequilibrações alcançam patamares cada vez mais elevados de conhecimento. Para Piaget (1977), os desequilíbrios desempenham o papel de "motor" da investigação e, ao ultrapassá-los, o sujeito pode repetir uma ação modificando-a via resultados, configurando assim uma "regulação" (processo de reação a uma perturbação).

O esforço operatório do sujeito (conjunto de ações reversíveis interiorizadas) envolve operações concretas, baseadas na percepção dos objetos (classificação e seriação) associadas às abstrações empíricas e as operações formais, as quais ocorrem sobre as reações do pensamento transformando os invariantes em abstrações aplicáveis a qualquer

3 Esquema é uma estrutura mental representativa dos aspectos do mundo, que além de organizar o conhecimento atual, serve de base para compreensões futuras. 
aspecto da realidade, em conjunto com as abstrações reflexionantes. Este caráter genérico das operações proposicionais apresenta-se combinadas como uma "rede" de associações ou através do grupo de quatro transformações INRC (PIAGET; INHELDER, 1976).

A construção da lógica da "rede" dá-se ao serem considerados os fatores $\mathrm{P}$ e Q em conjunto com $\sim \mathrm{P}$ (negação de $\mathrm{P}$ ) e $\sim \mathrm{Q}$ (negação de $\mathrm{Q}$ ), originando as quatro associações básicas: (P.Q), (P . Q $),(\sim P . Q),(\sim P . \sim Q)$ que combinadas "n por n" em uma tabelaverdade, apresentam 16 combinações hipotéticas restritas a um limite superior $(\sim \mathrm{P} . \sim \mathrm{Q})$ $\mathrm{V}(\sim \mathrm{P} . \mathrm{Q}) \mathrm{V}(\mathrm{P} . \sim \mathrm{Q}) \mathrm{V}(\mathrm{P} . \mathrm{Q})$ (todas são verdadeiras) e um limite inferior $\sim[(\sim \mathrm{P} . \sim \mathrm{Q}) \mathrm{V}$ $(\sim \mathrm{P} . \mathrm{Q}) \mathrm{V}(\mathrm{P} . \sim \mathrm{Q}) \mathrm{V}(\mathrm{P} . \mathrm{Q})]$ (todas são falsas). O sujeito do estádio formal pode construir cognitivamente as 16 combinações binárias mesmo não sendo capaz de explicitá-las (Piaget, 1978). A outra estrutura lógica do período formal apresenta uma integração entre os agrupamentos de classe e série, formando um sistema de dupla reversibilidade (inversão e reciprocidade) denominado de Grupo INRC.

Assim, a junção dos dois fatores $(\mathrm{P}, \mathrm{Q})$ pode ser representada como:

- Identidade: $\mathrm{I}=(\mathrm{P} V \mathrm{Q})$;

- Negação: $\mathrm{N}=(\sim \mathrm{P}$. Q), obtida invertendo a função e os valores dos fatores da identidade;

- Recíproca: $\mathrm{R}=(\sim \mathrm{P} \mathrm{V} \sim \mathrm{Q})$, obtida invertendo somente os valores dos fatores da identidade;

- Correlação: $\mathrm{C}=(\mathrm{P}$. Q), obtida invertendo a função e os valores dos fatores da recíproca;

A lógica destas estruturas foi empregada como estratégia de desenvolvimento do experimento virtual deste trabalho, no sentido do êxito ser alcançado por coordenações de ações (abstrações reflexionantes) condutoras de uma possível tomada de consciência e construção conceitual.

\section{MATERIAIS E MÉTODOS}

Este trabalho consiste em um estudo de caso qualitativo que busca entender as formas pelas quais os indivíduos representam suas experiências e interatuam em diferentes situações, dentro de um espaço definido de tempo. A coleta de dados inspirou-se no Método Clínico de Piaget (DELVAL, 2002) e ocorreu através da aplicação individual de uma entrevista clínica, de aproximadamente 30 minutos, a 10 estudantes do Ensino Médio, com base em um protocolo criado para sistematizar as atividades experimentais, desafiando o sujeito a explicar como a manipulação dos resistores R1 e R2 resulta no desligamento da lâmpada lp3. As atividades de simulação foram capturadas, em áudio e vídeo, da tela do computador utilizando o programa CamStudio (camstudio.org/). A análise foi realizada a partir dos estudos de Piaget e Inhelder (1976) sobre o pensamento da criança e do adolescente, buscando identificar nas respostas do sujeito elementos comprobatórios do esforço operacional ao longo da experimentação.

\subsection{Desenvolvimento do experimento no ambiente virtual}

Inspirado no uso de aparatos físicos para compreender a operatoriedade do pensamento (PIAGET E INHELDER, 1976), este artigo aborda o desenvolvimento e a aplicação de um experimento virtual imersivo de eletricidade, projetado para representar o funcionamento integrado dos dois tipos de reversibilidade (inversão e reciprocidade), 
propostos pela Epistemologia Genética. A escolha da plataforma OpenSim ${ }^{4}$, ocorreu pela disponibilidade gratuita e similaridade ao Second Life, o visualizador Singularity Viewer foi selecionado pela boa compatibilidade com a plataforma.

O experimento foi desenvolvido com base nos estudos do físico alemão Gustav Robert Kirchhoff (1824-1887) e aborda a conservação da carga elétrica ${ }^{5}$ mediante a convergência e divergência de correntes elétricas ${ }^{6}$ nos "nós" ${ }^{7}$ " de um circuito elétrico, fato conhecido como Primeira Lei de Kirchhoff (YOUNG E FREEDMAN, 2016).

Para este experimento, o circuito criado é do tipo paralelo, envolve diversos componentes elétricos e possui um total de dois (02) nós, três (03) $\operatorname{ramos}^{8}$ e três $(03)$ malhas ${ }^{9}$. A malha 01 localiza-se à esquerda do amperímetro central, enquanto a malha 02 está à direita do mesmo, havendo também uma malha 03 (externa). Para facilitar a análise, o nó superior foi identificado pela letra "A", o inferior pela letra "B" e foram determinadas as correntes $i_{1}, i_{2}$ e $i_{3}$ como as circulantes nas malhas. O sentido de circulação das correntes adotado é o convencional (do polo positivo para negativo da respectiva bateria). Como existe a possibilidade de modificação na posição das baterias (troca de orientação de polarização), podem ocorrer inversões no sentido de circulação das correntes. O diagrama esquemático pode ser visualizado na figura 01.

Figura 01: Esquema elétrico do experimento.

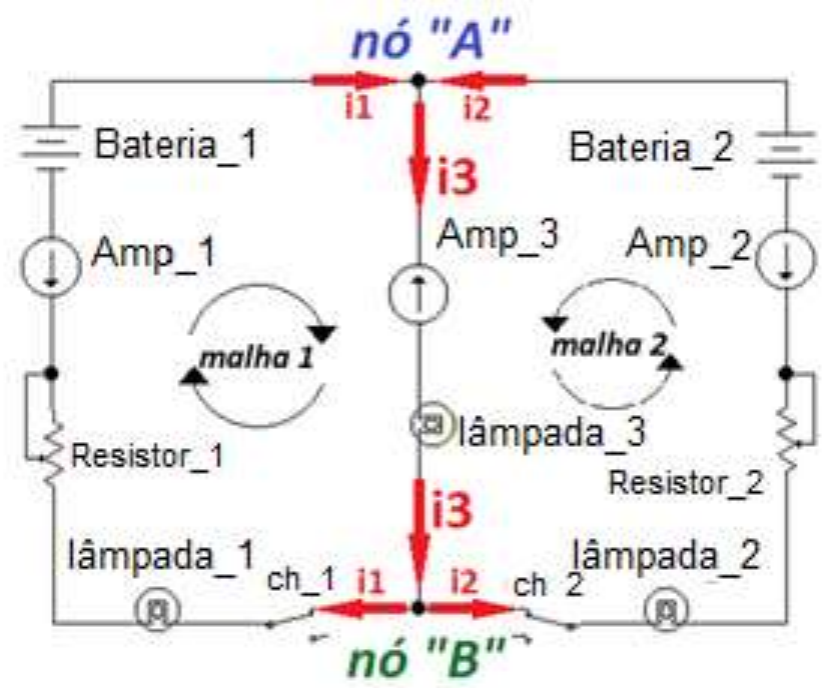

Fonte: o autor.

Análise do circuito proposto parte da premissa da impossibilidade física da criação ou desaparecimento das cargas elétricas em um nó, levando ao enunciado da Primeira Lei de Kirchhoff, "a soma algébrica das correntes é sempre igual a zero".

$$
\sum_{i=1}^{n} I_{i}=0 \text { (Eq.1) }
$$

Adotando a convenção de "positivas" para as correntes que entram e "negativas" para as oriundas do nó, tem-se a seguinte expressão:

$$
\sum_{i=1}^{n} I_{i_{\text {chegam }}}-\sum_{j=1}^{m} I_{j_{\text {saem }}}=0 \text { (Eq.2) }
$$

\footnotetext{
${ }^{4}$ OpenSimulator, frequentemente chamado de OpenSim, é um servidor open source para hospedagem de mundos virtuais similar ao Second Life (http://opensimulator.org.).

${ }^{5}$ Conservação da carga elétrica é o princípio da Física que estipula a impossibilidade de criação ou destruição de cargas elétricas.

${ }^{6}$ Movimento ordenado de cargas elétricas em um condutor, em um determinado sentido e ocasionado por uma diferença de potencial elétrico.

${ }^{7}$ Entende-se por nó, o ponto de encontro de três ou mais ramos de um circuito, tendo cada ramo um ou mais componentes elétricos.

${ }^{8}$ Ramo: é o trajeto entre dois nós consecutivos, pelo qual circula uma corrente elétrica de valor constante.

${ }^{9}$ Malha: é um trecho de circuito que forma uma trajetória eletricamente fechada.
} 
Passa-se então, o segundo termo para o outro lado da igualdade:

$$
\sum_{i=1}^{n} I_{i_{\text {chegam }}}=\sum_{j=1}^{m} I_{j_{\text {saem }}}(\text { Eq.3) }
$$

Dessa forma, é possível concluir que o somatório das correntes incidentes é igual ao das provenientes do nó, aplicando tal conclusão aos nós do circuito da figura 01, têm-se:

$$
\begin{array}{ll}
\text { Nó } A: i_{1}+i_{2}-i_{3}=0 \text { (Eq.4) } & i_{3}=i_{1}+i_{2} \text { (Eq.5) } \\
\text { Nó B: }-i_{1}-i_{2}+i_{3}=0 \text { (Eq.6) } & i_{3}=i_{1}+i_{2} \text { (Eq.7) }
\end{array}
$$

A comparação entre as equações extraídas dos nós A e B revela uma igualdade entre as mesmas, pois o número de equações independentes obtidas pela aplicação da lei das correntes é sempre igual ao número de nós do circuito, menos um. Como consequência, somente uma equação é suficiente para entender o comportamento das correntes no circuito.

No tocante ao experimento virtual (figura 02), este foi projetado em consonância com a possibilidade do sujeito converter ações progressivas e reversíveis em operações cognitivas (PIAGET, 1976).

Figura 02: Experimento virtual imersivo 3D sobre a $1^{\text {a }}$ Lei de Kirchoff.

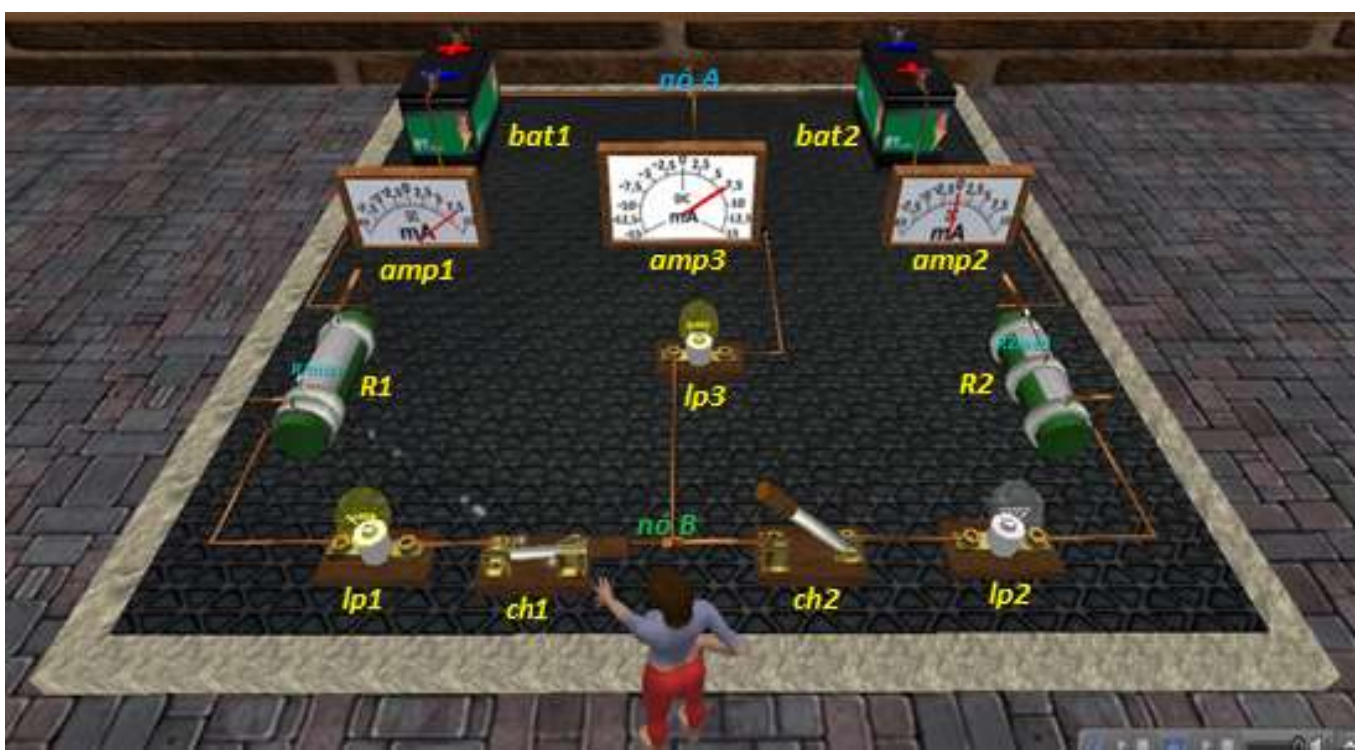

Fonte: o autor.

As transformações disponibilizadas para o sujeito modificar o experimento e assimilálo enquanto objeto do conhecimento são as seguintes:

a) Abertura ou fechamento dos interruptores ch1 e ch2, controlando a passagem de corrente elétrica pelo circuito;

b) Aumento ou diminuição do valor da resistência elétrica dos resistores R1 e R2, através da manipulação do terminal central;

c) Ampliação ou decréscimo do valor das correntes elétricas nas malhas, pela modificação da posição do terminal central dos resistores R1 e R2;

d) Inversão na polaridade das Baterias 01 e 02 , pela em alteração em $180^{\circ}$ da posição horizontal das mesmas;

No experimento, quando as baterias estiverem em "contra-fase" (polaridades invertidas entre si) o módulo de $i_{3}$ (corrente no ramo central) será a diferença entre $i_{1}$ e $i_{2}$, e o sentido de $i_{3}$ determinado pela corrente de maior valor $\left(i_{1}\right.$ ou $\left.i_{2}\right)$, isto pode ser visualizado na figura 03 . 
Figura 03: Corrente elétrica no ramo central para baterias em contra-fase.

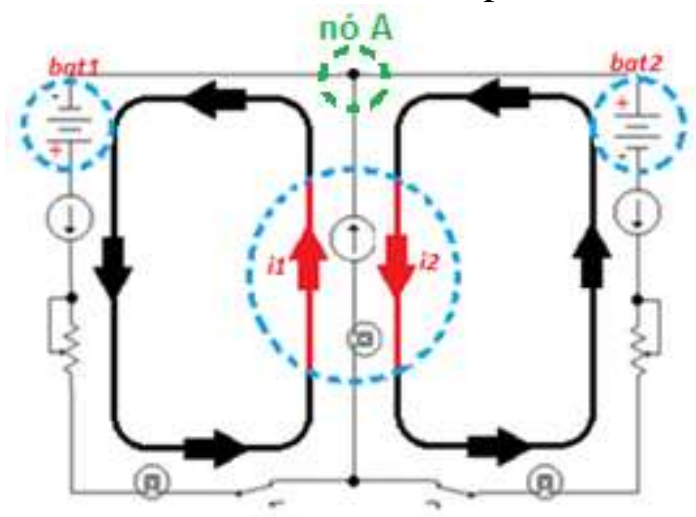

Fonte: o autor.

O equilíbrio de correntes no ramo central é alcançado quando $i_{1}$ e $i_{2}$ possuem o mesmo módulo (R1 e R2 com a mesma resistência elétrica), mas sentidos inversos (Bat1 e Bat2 com polaridades antagônicas). O resultado é uma corrente nula $\left(i_{3}=0\right)$ que apaga a lâmpada do ramo central (lp3), mesmo com ambos interruptores (ch1 e ch2) fechados. Neste circuito, para qualquer outra situação (a não ser ch1 e ch2 abertos) a lâmpada lp3 ficará acesa (mesmo que minimamente) indicando um desequilíbrio entre $i_{1}$ e $i_{2}$, cujo valor será mostrado pelo ponteiro do amperímetro central.

Para constituir um conjunto de operações reversíveis e integradas em um único sistema, trabalhou-se com a situação de equilíbrio entre as correntes $i_{1}, i_{2}$ e $i_{3}$ circulando pelo nó "A" do circuito.

A integração das seguintes transformações compõe um sistema de dupla reversibilidade em uma situação de equilíbrio:

I. DIRETA: ação sobre o valor da corrente elétrica ( $i_{1}$ ou $\left.i_{2}\right)$ de uma das malhas (incremento de magnitude);

II. INVERSA: negação da ação anterior (decremento do valor da corrente elétrica $\mathrm{i}_{1}$ ou $\mathrm{i}_{2}$ );

III. RECIPROCIDADE: compensação entre as correntes elétricas $i_{1}$ e $i_{2}$, ocorrida no ramo central do circuito (possibilidade de anulação da resultante $i_{3}$ devido a $i_{1}$ e $i_{2}$ possuírem mesmo valor, mas sentidos opostos);

IV. CORRELAÇÃO: negação da ação anterior pela soma dos valores das correntes no ramo central (no caso das baterias 1 e 2 estarem polarizadas no mesmo sentido);

A partir destas transformações, as operações proposicionais para constituir o grupo INRC são:

1) $I\left(i_{a} V i_{b}\right)=$ operação direta: representa dois valores diferentes e arbitrários de corrente elétrica na mesma malha, determinados pelo respectivo valor da resistência elétrica de $\mathrm{R} 1$ ou $\mathrm{R} 2$.

2) $\mathrm{N}\left(\sim \mathrm{i}_{\mathrm{a}} \bullet \sim \mathrm{i}_{\mathrm{b}}\right)=$ operação inversa: representa a supressão de $\mathrm{i}_{\mathrm{a}}$ ou $\mathrm{i}_{\mathrm{b}}$ pela variação, em sentido contrário ao item "1)", da resistência elétrica de R1 ou R2 (se a corrente anterior aumentou, agora é diminuída e vice-versa);

1) $\mathrm{R}\left(\sim \mathrm{i}_{\mathrm{a}} \mathrm{V} \sim \mathrm{i}_{\mathrm{b}}\right)=$ operação recíproca: compensação no ramo central de $\mathrm{i}_{1}$ por $\mathrm{i}_{2}$ e vice-versa, devido a possuírem sentidos inversos de circulação. 
2) $\mathrm{C}\left(\mathrm{i}_{\mathrm{a}} \bullet \mathrm{i}_{\mathrm{b}}\right)=$ operação correlativa: negação da recíproca (anulação dos efeitos contrários desta). Aplicada ao caso das baterias estarem com a polaridade no mesmo sentido, de modo somar os valores das correntes $i_{1}$ e $i_{2}$ no ramo central.

\section{RESULTADOS E DISCUSSÃO}

O experimento foi aplicado a 10 sujeitos, com variadas idades e cursando ensino médio ou graduação, a simulação durou aproximadamente 30 minutos e foi realizada utilizando o próprio computador do investigador, não foi necessário o emprego da internet, pois o ambiente virtual estava instalado no modo Standalone. Os diálogos foram transcritos em forma de protocolo e categorizados para identificar elementos indicativos do esforço operacional cognitivo realizado pelos sujeitos.

Quanto aos aspectos lógico-matemáticos do esforço cognitivo foi observado uma evolução nos esquemas de ação dos sujeitos, pois se no início, apresentaram alguma dificuldade operar o experimento, em seguida estavam testando hipóteses e respondendo corretamente aos questionamentos das situações-problema colocadas pelo entrevistador, como pode ser visto a seguir:

TON $(18 ; 3)$ "O que acontece no circuito se for acionado o interruptor da esquerda?" Não soube dizer com certeza o que ocorreria, nem conseguiu identificar o circuito como um caminho fechado para a corrente elétrica, pois para ele, somente acenderia a lâmpada da esquerda (deveriam acender a da esquerda e a central).

TON $(18 ; 3)$ "Considerando o circuito da esquerda com corrente de $+2,5 \mathrm{~mA}$, o da direita com corrente de $-2,5 \mathrm{~mA}$, as lâmpadas esquerda e direita acesas e a do centro apagada. Qual será o comportamento do circuito, caso o valor do resistor da direita for alterado do máximo para o seu valor médio?" O sujeito foi refletindo e antecipando corretamente as situações que iriam ocorrer, respondendo: "a corrente vai ficar mais negativa, a lâmpada da esquerda vai acender forte (amarela), a do centro vai acender fraco (filamento avermelhado) e a da esquerda não vai se alterar". A partir deste ponto, o sujeito continuou fazendo uma relação correta entre os valores mostrados no amperímetro e antecipar corretamente quando a lâmpada do ramo central estaria apagada.

No que concerne às abstrações reflexionantes, elas ocorreram ao longo das simulações, indicando a capacidade dos sujeitos de refletir sobre suas ações.

AMA $(19 ; 1)$ "O que deve ser feito para que a lâmpada do meio apague e as lâmpadas da direita e da esquerda permaneçam acesas?" O sujeito respondeu "as baterias têm de estar diferentes (referindo-se as polaridades) e os resistores têm que estar iguais (mesmo valor ôhmico)". Isso leva a crer que o sujeito, comparou o funcionamento dos circuitos da esquerda e direita e criou o conceito da lâmpada central ser desligada pelo somatório dos valores mostrados nos amperímetros.

\section{CONCLUSÕES}

De acordo com análise das simulações, as baterias e as lâmpadas foram facilmente identificadas, por outro lado, os interruptores foram confundidos com alavancas, os amperímetros com voltímetros e os resistores não foram identificados, denotando a ausência da prática experimental de circuitos elétricos. $\mathrm{O}$ dimensionamento do circuito obteve sucesso no quesito de "ações reversíveis internalizadas, transformarem-se em operações", pois grande parte dos sujeitos conseguiu solucionar mentalmente as situações-problema propostas, mostrando uma capacidade operatória relacionada com as ações passíveis de serem realizadas sobre o circuito. $\mathrm{O}$ experimento ofereceu condições 
de execução de regulações ativas, quando o indivíduo não possui uma resposta imediata para um questionamento e necessita executar várias ações repetidas e reversíveis sobre o circuito, para obter a solução desejada.

Não houve demonstração de conhecimento sobre a necessidade de um circuito fechado para a circulação de corrente, pois se os sujeitos tivessem construído tal conceito deveriam responder, por exemplo, que ao ser acionado o interruptor da esquerda acenderiam as lâmpadas da esquerda e do centro, com os respectivos amperímetros apresentando o mesmo valor de corrente elétrica. Os sujeitos também não demonstraram conhecimento sobre o conceito de corrente elétrica ou quais grandezas físicas presentes no circuito a influenciam. Não foi identificada uma relação entre a alteração da posição (polaridade) da bateria com o sentido de circulação de corrente elétrica, demostrando que, talvez, este conceito ainda não tenha sido construído.

No entanto, os sujeitos conseguiram, por si só, detectar a independência entre os ramos da esquerda e da direita, devido a observação dos valores mostrados pelos amperímetros. Muito embora tenham conseguindo uma experiência frutífera relativa a apropriação da lógica operacional do experimento, não foi possível verificar uma "lógica física" que representasse a interconexão correta entre os conceitos de resistência, corrente e voltagem elétricas.

Os sujeitos pareceram muito ligados às características observáveis do experimento sem associar a lógica do circuito às leis e conceitos físicos que o regem, indicando deficiências na aprendizagem de eletricidade. Uma possível solução está sendo implementada através do desenvolvimento de um circuito mais simples, capaz de vincular suas operações com os conceitos básicos de voltagem, corrente e resistência elétricas. Com isso, espera-se gerenciar a complexidade do circuito atual apresentando antes, uma outra simulação de caráter preparatório.

Com relação ao esforço operatório, houve o surgimento de evidências de operações concretas como classificação e seriação, além de operações formais relativas a lógica em rede, com base em evidências do uso de teorização sobre os resultados do experimento. Neste momento o experimento não logrou o êxito esperado em verificar operações de dupla reversibilidade relacionadas ao grupo INRC (fato que parece estar ligado a complexidade do circuito apresentado). Como alternativa está sendo desenvolvido um circuito de menor complexidade para ser apresentado antes do circuito principal, com vistas a qualificar o processo de equilibração dos estudantes.

\section{REFERÊNCIAS BIBLIOGRÁFICAS}

BRASIL. Ministério da Educação. Secretaria de educação básica. PCN+ Ensino Médio

- Física. Orientações Educacionais Complementares aos Parâmetros Curriculares Nacionais. Brasília: MEC/SEMTEC, 2006.

DELVAL, J. Introdução à Prática do Método Clínico, Ed. Artmed, 2002.

GUEDES, L. D. dos S. Experimentos com materiais alternativos: sugestão para dinamizar a aprendizagem de eletromagnetismo. Dissertação de Mestrado Universidade Federal de Goiás, Unidade Acadêmica Especial de Física e Química, Catalão, Programa de Pós-Graduação em Ensino de Física, Catalão, 2017. 
HALLIDAY, D., RESNICK, R., WALKER, J. - Fundamentos de Física 3 - 10a edição - Livros Técnicos e Científicos Editora S/A (LTC) - Rio de Janeiro, 2016.

MENTZ, L. O uso da pesquisa para o ensino das ondas eletromagnéticas. Dissertação de Mestrado. Instituto de Física da Universidade Federal do Rio Grande do Sul, Porto Alegre: IF, 2017.

MORAES, C. J. C. A apropriação da teoria de Jean Piaget no Ensino de Ciências. Dissertação (Mestrado) - Universidade Federal de Goiás, Faculdade de Educação, Programa de Pós-Graduação em Educação, Goiânia, 2017.

OBANA, J.E.G. A epistemologia e a psicologia de Jean Piaget e as neurociências uma revisão sistemática. Dissertação de Mestrado, Universidade estadual paulista Júlio de Mesquita Filho, Programa de Pós-graduação em Educação, Marília, SP, 2015.

PIAGET, J.; INHELDER, B. Da lógica da criança à lógica do adolescente, Livraria Pioneira Editora, São Paulo, 1976.

PIAGET, J. A tomada de consciência, Melhoramentos, Ed. da Universidade de São Paulo, São Paulo,1977.

PIAGET, J. Fazer e compreender, Edições Melhoramentos- Editora da USP, São Paulo, 1978.

SANTOS, J. V. C. Aprendizagem do eletromagnetismo em cursos de engenharia: uma proposta da construção de laboratório virtual com abordagem de campos conceituais. Dissertação de Mestrado - Universidade do Estado da Bahia. Departamento de Educação. Mestrado Profissional Gestão e Tecnologias Aplicadas a Educação Salvador, 2016.

YOUNG, H. D.; FREEDMAN, R. A. FÍSICA III - Eletromagnetismo. São Paulo: Pearson, 2016. 